\title{
Wirksamkeitsforschung im Führungskräfte-Coaching
}

\author{
Hansjörg Künzli
}

Zusammenfassung: Im Artikel wird Bezug genommen auf die erste Übersichtsarbeit zum Thema Wirksamkeitsforschung im Führungskräfte Coaching (vgl. OSC 12, 3, 2005). Darüber hinaus werden acht neue Arbeiten zum Thema vorgestellt. Der Stand der Forschung wird kritisch hinterfragt, und es werden neue Forschungsfragen aufgezeigt. Obwohl die Anzahl der empirischen Studien immer noch gering ist und die meisten mit methodischen Mängeln behaftet sind, entsteht der Eindruck, dass Coaching wirkt. Als kritische Größe für zukünftige Forschung wird der Zugang zum Feld betrachtet. Dieser ist nach wie vor schwierig. Ein Grund dafür wird darin gesehen, dass die herkömmliche, auf durchschnittliche und nicht individuelle Veränderung fokussierte Forschung von Praktikern als wenig hilfreich oder nützlich wahrgenommen wird. Entsprechend wird für eine klientenorientierte Forschung plädiert.

Schlüsselwörter: Führungskräfte-Coaching $\cdot$ Wirksamkeitsforschung $\cdot$ Evaluation

\section{Outcome research on executive coaching}

\begin{abstract}
In the article the author refers to his first paper reviewing 22 empirical studies in the field of executive coaching (cf. OSC 12, 3, 2005). Furthermore eight new studies are presented and existing research is critically analyzed. As a result implications for further research are suggested. Though the body of empirical research is still sparse and most of the reported studies had severe limitations, they provide evidence that coaching works. Access to the field of executive coaching is considered difficult for researchers but will be crucial for future research. It is hypothesized that conventional research focusing on average and not on individual changes is perceived neither helpful nor useful by practitioners. To meet practitioners' needs, a client focused research is advocated.
\end{abstract}

Keywords: Executive coaching $\cdot$ Outcome research $\cdot$ Evaluation

(C) VS-Verlag 2009

Prof. H. Künzli $(\bowtie)$

Zürcher Hochschule für Angewandte Psychologie, Minervastrasse 30, 8032 Zürich, Schweiz

E-Mail: hansjoerg.kuenzli@zhaw.ch

www.psychologie.zhaw.ch 


\section{Einleitung}

Vor vier Jahren durfte ich an gleicher Stelle einen Artikel zum Stand der Dinge der Wirksamkeitsforschung im Führungskräfte-Coaching (Künzli 2005) veröffentlichen. Damals stellte ich einen bemerkenswerten Mangel an wissenschaftlichen Arbeiten zum Thema fest. Seither hat sich die Situation zwar verbessert, doch überwältigend ist die Anzahl der Arbeiten noch keineswegs. Dies steht ganz im Gegensatz zum allgemeinen Interesse am Thema: Nimmt man die Einträge im Internet als Vergleichsgröße, würde Coaching in einem Beliebtheitsranking psychologischer Beratungssettings ganz weit oben stehen. Während der Begriff ,Coaching' in einer Google-Recherche (Stand Dezember 2008) fast 70 Millionen Treffer zutage fördert und Supervision mehr als 42 Millionen, bringt es die Psychotherapie gerade mal auf ca. 6 Millionen.

Doch die Zeit ist nicht stehengeblieben. In den letzten Jahren hat sich die Wissenschaft vermehrt des Themas angenommen, und die Anzahl einschlägiger Publikationen, seien diese nun theoretischer oder empirischer Art, ist eindeutig gestiegen. Neben der regelmäßig aktualisierten Literaturübersicht von Rauen (http:/www.coaching-literatur.de), den Übersichtsarbeiten von Grant $(2001,2005)$ und seiner Sammlung englischsprachiger Abstracts (2007) sind vier wissenschaftliche Zeitschriften erschienen, die sich des Themas annehmen: Das International Journal of Evidence Based Coaching and Mentoring (Ersterscheinungsjahr 2003); The Coaching Psychologist (Ersterscheinungsjahr 2005); International Coaching Psychology Review (Ersterscheinungsjahr 2006), und Coaching: An International Journal of Theory, Research and Practice (Ersterscheinungsjahr 2008).

Einen Meilenstein der wissenschaftlichen Auseinandersetzung mit Coaching stellt das Buch „Coaching und ergebnisorientierte Selbstreflexion“ von Greif (2008) dar, welches u.a. eine Übersicht zum Stand der Evaluationsforschung im Coaching beinhaltet. Greif analysiert die Arbeiten und entwirft ein Strukturmodell der Coachingwirkungen mit den Elementen Voraussetzungen (für Wirkungen), Wirkfaktoren und Ergebnisse von Coaching. Die Voraussetzungen werden unterteilt in Coach- und Klientenvariablen, die Ergebnisse in spezifische und allgemeine. Da eine empirische Überprüfung noch aussteht, bezeichnet Greif (2008: 275) das Modell als vorläufige Orientierungsgrundlage. Das Modell zeigt im Übrigen große Ähnlichkeit mit dem Generic Modell of Psychotherapy (Orlinsky \& Howard 1986), was als Vorteil zu werten ist. Denn es zeigt, dass bei aller Unterschiedlichkeit hinsichtlich Zielgruppen, Kontext und Zielen auf struktureller Ebene durchaus sehr viel Vergleichbarkeit besteht. Für eine zukünftige, interdisziplinär ausgerichtete Forschung kann sich die Anlehnung an die Psychotherapieforschung als fruchtbar erweisen.

Zwecks Vergleichbarkeit mit der ersten Publikation wurden nur empirische Arbeiten mit Führungskräften, die sich der Wirksamkeit, d.h. den Wirkungen und den Wirkfaktoren von Coaching widmen, rezipiert. Ausgeschlossen wurden z. B. Befragungen von Coaches zu den Themen Wirkungen und Wirkfaktoren (z. B. Hess \& Roth 2001; Befragung von Coaches zu Struktur-, Prozess- und Ergebnisqualität). Nicht berücksichtigt wurden auch Arbeiten mit anderen Zielgruppen als Führungskräfte (z. B. Coaching von Mitarbeitenden durch Führungskräfte oder Mitarbeitende, vgl. z. B. Olivero et al. 1997; Life-Coaching von Personen ohne Führungsfunktion, vgl. z. B. Grant 2003; Gesundheitscoaching außerhalb des Führungskontextes, Grant 2008; Green, Oades \& Grant 2005; 
Spence \& Grant 2005) und Einzelfallstudien (eine Übersicht zu diesem Thema findet man bei Grant 2001). Zwei Arbeiten (Hernez-Broome 2004; Dingman 2004) erfüllten zwar die Kriterien, waren aber nicht verfügbar.

Um der Vollständigkeit willen werden die bei Greif (2008: 212 ff.) besprochenen Studien genannt, aber nicht besprochen, um Redundanz zu vermeiden. Es handelt sich dabei um Arbeiten, die mir in der ersten Übersicht entweder entgangen sind (von Bose et al. 2003; Böning \& Fritschle 2005; Dzierzon 2004; Runde \& Bastians 2005; Sauer et al. 2004; Steinmetz 2005; Sue-Chan \& Latham 2004), oder zum Zeitpunkt der Abgabe des Manuskripts noch nicht verfügbar waren (Finn-Mason \& Griffin 2006; Kaufel et al. 2006; Mellmann 2006). Trotz der gestiegenen Beschäftigung mit dem Thema ist die absolute Anzahl der Arbeiten noch sehr gering. Vorherrschend sind nach wie vor Konzept- und Theoriearbeiten. Während meine erste Recherche 22 den Einschlusskriterien genügende Artikel zutage förderte (Künzli 2005), sind es nun 40, was einem Zuwachs von 18 Arbeiten entspricht. 10 davon werden eingehend bei Greif besprochen, 8 sind Gegenstand des vorliegenden Artikels. Wiederum beschränkte sich die Recherche nicht auf Peer Reviewed Journals. Willkommen waren auch Belege aus der grauen Literatur, die zwar nicht unbedingt den Standards der Wissenschaftlichkeit genügen, aber sinnvolle Resultate produzieren.

\section{Die Studien}

(1) Auf dem Hintergrund der Tatsache, dass Vertrauen zwischen Coach und Klient in der einschlägigen Literatur als Schlüsselvariable für positive Ergebnisse bezeichnet wird, stellen sich Alvey \& Barcley (2007) die Frage, wie sich Vertrauen zwischen Coach und Klient entwickelt. In der qualitativ angelegten Studie mit 27 Führungskräften entwickeln die Autoren eine Theorie der Vertrauensentwicklung zwischen Coach und Coachee. Relationale, situative und verhaltensbezogene Faktoren beeinflussen die Vertrauensentwicklung. Am höchsten war das Vertrauen, wenn der Klient (1) willens war, offen und ehrlich über seine Gefühle und Gedanken zu sprechen, und er gleichzeitig eine unterstützende und nicht wertende Reaktion vom Coach erhielt, (2) die Organisation sich gegenüber dem Coaching offen und unterstützend zeigte, (3) Coach und Klient sich im Klaren waren hinsichtlich der Erwartungen bezüglich Vertraulichkeit und der zu erzielende Ergebnisse und (4) der Coach unterstützend und bestätigend auf die Entwicklungsbedürfnisse des Klienten das Führungsverhalten des Klienten hinterfragt.

(2) Anderson (2004) und Anderson, Brill \& Lynch (2007) aus den Beratungsunternehmen MetrixGlobal LLC und Linkage Inc. beschäftigen sich mit der Schätzung des monetären Ertrags von Personalentwicklungsmaßnahmen, insbesondere von Coaching. Anderson (2004) interviewte Führungskräfte mit eigenen Coachingerfahrungen und schätzte den Return of Investment auf 689\%. In einer weiteren Publikation (Anderson, Brill \& Lynch 2007) werden 83 Führungskräfte einige Zeit nach ihrem Coaching gebeten, das Ausmaß der direkt auf die Coachingmaßnahme zurückzuführenden Produktivitätszunahme sowie die Zunahme des Nettoertrags in Prozenten zu schätzen. Die durchschnittliche geschätzte Zunahme der Produktivität betrug 6,3\%, diejenige der des Nettoertrags 6,5\%. Danach wurde die Lohnsumme der 83 Führungskräfte (insgesamt 18,7 Mio. \$) 
mit der Produktivitätszunahme multipliziert, was einen Wert von 1,365 Mio. \$ für den Wert der Produktivitätszunahme ergab. In einem zweiten Schritt addierten die Autoren die prozentuale Zunahme des Nettoertrags (6,5\%) mit den Nettoeinnahmen (113 Mio. $\$$ ) und addierten diesen Betrag (7 Mio.) zum Wert der Produktivitätszunahme, was zu einen Gesamtwert von 8.365 Mio. \$ führt. Um eine konservative Schätzung zu erhalten, reduzierten sie diesen Wert um die Hälfte und beziffern den Wert des Coachings mit gut 4 Mio. \$. Zweifelhaft an diesem Vorgehen sind die Verlässlichkeit einer solche Schätzung und die kausale Verbindung mit der Maßnahme.

(3) Bowles et al. (2007) untersuchten Zielerreichung, den Zuwachs von neun Führungskompetenzen und den Rekrutierungserfolg von insgesamt 59 Rekrutierungsmanagern (30 mittleres Management, 29 höheres Management) der US-Armee. Wobei beide Führungsstufen nicht mehr direkt in die Rekrutierungsprozess involviert waren, sondern so genannte „Station Commanders“ mit einer Anzahl direkt unterstellten Rekrutierungsbeamter und so genannte „Company Commanders“ als Vorgesetzte von mehreren „Station Commanders“. Das Programm war auf 12 Monate ausgelegt. Ziele konnten individuell gewählt werden. Alle Coaches hatten viel Erfahrung in der Rekrutierung. Die mittleren Manager nahmen durchschnittlich 405', die höheren Führungskräfte 420' Coaching in Anspruch. Anzahl und Dauer der Treffen wurde individuell vereinbart. Jede Führungskraft konnte bis zu fünf frei gewählte Ziele bearbeiten. Die gewählten Ziele wurden nachträglich den drei Bereichen Rekrutierung, Lebensqualität und Führung zugeordnet.

Als wichtige Größe für den Coaching-Erfolg betrachteten die Autoren das Engagement der Klienten. Die Einschätzung des Engagements erfolgte durch den Coach auf einer 3Punkte-Skala (verschlossen/wenig empfänglich für Inputs; einigermaßen empfänglich für Inputs; nimmt Rat und Unterstützung offen entgegen/empfänglich für Input) und wurde nach jeder Sitzung und am Ende des Coachings erhoben. Des Weiteren schätzten die Coaches den individuellen Lernzuwachs ihrer Klienten, wiederum auf Dreipunktskalen, auf neun für ihre Aufgabe relevanten Kompetenzen ein. Jede der drei Ausprägungen pro Kompetenz wurde mit einem im Verhalten verankerten Beispiel beschrieben.

Die Manager der beiden Führungsstufen unterschieden sich nicht im Ausmaß, aber in der Art der gewählten Ziele. Während beide Gruppen im Schnitt praktisch die volle Anzahl möglicher Ziele (4.8 vs. 4.7) bearbeiteten, wählten die Führungskräfte der mittleren Stufe am meisten Ziele aus dem Bereich Lebensqualität, gefolgt von Leistung und Führung. Die höheren Führungskräfte bearbeiteten am meisten Ziele aus dem Leistungsbereich, gefolgt von solchen aus den Bereichen Lebensqualität und Führung. In der Gruppe mittleres Management korrelierte das Coaching-Engagement signifikant mit dem Lernfortschritt in den Kompetenzen Zeitmanagement und direkte Führung der Mitarbeitenden. In der Gruppe der höheren Führungskräfte wurden drei signifikante Korrelationen gefunden: Direkte Führung der Mitarbeitenden, analytische Fähigkeiten und Leadership (z.B. klare Kommunikation, Einschätzung der Fähigkeiten der Mitarbeitenden, etc.). Kein Zusammenhang wurde gefunden zwischen dem Coaching-Engagement und der Rekrutierungsleistung.

Das interessanteste Ergebnis betraf die Rekrutierungsleistung. Im Referenzjahr übertrafen beide Gruppen der gecoachten Führungskräfte die Leistungen einer Vergleichsgruppe erfahrener, aber nicht am Programm teilnehmender Führungskräfte signifikant, wobei die Gruppe der Teilnehmer aus der mittleren Führungsebene am meisten profitierte. 
Ein zweiter Vergleich der Interventionsgruppen mit dem nationalen Leistungsschnitt des Referenzjahres zeigte eine signifikante Mehrleistung der mittleren Manager, nicht aber der höheren Führungskräfte. Eine Hochrechnung der Mehrleistung auf Ebene mittleres Management auf die ganze USA ergab ein Plus von 11'200 (10,2\%) zusätzlichen Rekrutierungen, was die Autoren als beträchtliche Leistungen bezeichnen. Bemerkenswert ist, dass die zusätzliche Leistung nicht direkt, sondern indirekt über die Rekrutierungsbeamten zustande kam.

(4) Evers et al. (2006) untersuchen die Wirkungen von Coaching in einer quasiexperimentellen Studie mit jeweils 30 Personen in der Kontroll- und der Versuchsgruppe. Rekrutiert wurden die Teilnehmenden der Versuchsgruppe aus 41 Managern aus verschiedenen Departements der öffentlichen Verwaltung, die beabsichtigten, ein Coaching zu beziehen. Die Kontrollgruppe wurde aus einer Auswahl von 77 Managern des Departements Stadtentwicklung gebildet. Versuchs- und Kontrollgruppe wurden, so gut es ging, anhand der Variablen Gehalt, Geschlecht und Alter gematched. Die Untersuchungsdauer betrug vier Monate. Die Teilnehmenden nahmen durchschnittlich an 3,7 Sitzungen (min. 1 bis max. 8) teil. Die Teilnehmenden füllten das Erhebungsinstrument direkt vor und ein zweites Mal vier Monate später nach dem Coaching aus.

Ausgangspunkt der Einzelcoachings mit individuellen Zielsetzungen war Whitmore's GROW-Modell (2002), wobei G für ,goal setting“, R für „reality“, O für „options“ und W für „,will power“ steht. Der Coach hilft dem Klienten bei der Zielklärung und -konkretisierung $(G)$, unterstützt ihn dabei, die Ziele so zu setzen, dass sie realisierbar sind (R), erarbeitet mit ihm zusammen die besten Wege und Optionen zur Zielerreichung $(\mathrm{O})$ und begleitet ihn bei der Handlungsausführung (W). Während des Coachings wurden verschiedene Methoden wie z.B. Rollenspiele, rational-emotives Training, Brainstorming und solche zur Zielsetzung eingesetzt.

Der Erfolg des Coachings wurde mit einem selbst entwickelten Fragebogen mit 35 10stufigen Items und 6 Dimensionen erfasst. Die Konstruktion der 6 Dimensionen erfolgte in Anlehnung an Banduras (1986) Konzepte der Selbstwirksamkeits- und der Ergebniserwartungen. Beide Erwartungstypen wurden in Bezug gesetzt zu drei auf dem Modell von Withworth (1998) basierenden handlungsrelevanten Bereichen: Zielsetzung, balanciertes, ausgewogenes Handeln und Achtsamkeit. Dies führte zu einem Instrument mit insgesamt sechs Dimensionen. Auffällig ist, dass sich alle Dimensionen bei beiden Gruppen in unterschiedlichem Ausmaß in die positive Richtung entwickeln. Signifikante Unterschiede zwischen der Versuchs- und der Kontrollgruppe sind aber nur auf den Dimensionen Ergebniserwartung bezüglich balanciertem, ausgeglichenem Handeln und der zielbezogenen Selbstwirksamkeitserwartung zu verzeichnen. Schwierig zu interpretieren ist die Zunahme der Ergebniserwartung, die sich auf das balancierte Handeln bezieht. Selbstwirksamkeits- und Ergebniserwartung wurden von Bandura (1977: 193) als aufeinander folgend beschrieben. Während sich Selbstwirksamkeit auf die Handlungsausführung bezieht, richtet sich die Ergebniserwartungen auf die Annahme, dass eine Handlung ein bestimmtes Ergebnis nach sich zieht. Zu erwarten wäre daher, dass sich zunächst die Selbstwirksamkeit und dann in Abhängigkeit von ihr die Ergebniserwartung aufbauen müssten. So wie das Resultat vorliegt, ist es mithin schwer zu interpretieren.

(5) Gyllensten \& Palmer (2005) untersuchen die Wirkung von Coaching auf Stress, Ängstlichkeit und Depressivität mit einer quasi-experimentellen Studie. Die Teilneh- 
menden waren Angestellte eines mittelgroßen englischen Finanzdienstleisters. Unklar bleibt, ob es sich bei den Teilnehmenden um Angestellte mit oder ohne Führungsfunktion handelte. An der Studie nahmen 17 Männer und 15 Frauen teil. 16 nahmen am Coaching teil, 15 lieferten die Daten für die Kontrollgruppe. Die Studie dauerte 8 Monate. Die Teilnahme war freiwillig. Durchschnittlich nahmen die Personen der Versuchsgruppe an 3,8 Sitzungen teil (Minimum 1 Sitzung, Maximum 10 Sitzungen).

Das Belastungsniveau wurde mit dem DASS-21 (Lovibond \& Lovibond 1995) mit den drei Skalen Depressivität, Ängstlichkeit und Stress erhoben. Der DASS-21 gilt als valides und reliables Instrument zur Erfassung der erwähnten Dimensionen in nicht klinischen Populationen.

Entgegen der Erwartung wurden weder signifikante Interaktionen (unterschiedliche Verläufe der Gruppen) noch Haupteffekte (Veränderungen der Gesamtgruppe) auf den drei Zieldimensionen festgestellt. Die Depressivität nahm zwar bei der Kontroll- und der Versuchgruppe ab, aber ungefähr im gleichen Ausmaß. Zudem wurde die Veränderung nicht signifikant. Ein wenig anders sehen die grafischen Verläufe von Ängstlichkeit und der Stressbelastung aus. Während die Ängstlichkeit in der Kontrollgruppe sogar ein wenig zunahm, sank sie in der Versuchsgruppe deutlich, aber nicht signifikant ab. Die Stressbelastung nahm in beiden Gruppen ab, aber deutlicher innerhalb der Versuchsgruppe. Keines der Ergebnisse ist signifikant. Trotzdem wurde die Effektivität der Intervention mit durchschnittlich 6.4 auf einer 7-stufigen Skala sehr hoch eingeschätzt. Die Skalen Stress und Ängstlichkeit zeigten zwar einen Verlauf in die erwartete Richtung, die Veränderungen waren aber nicht signifikant. Das mag an den kleinen Stichproben liegen. Des Weiteren wissen wir wenig über die spezifische Wirksamkeit von Coaching im Zusammenhang mit den erwähnten Zielgrößen. Auch wenn die Ergebnisse nicht den Erwartungen entsprechen, ist die Untersuchung von Bedeutung. Zum einem, weil sie sich der psychischen Gesundheit widmet, einem für Coaching zunehmend wichtigen Thema, und zum anderen aufgrund der Verwendung einer Kontrollgruppe. Jede Intervention sollte ihre Überlegenheit gegenüber der Nicht-Intervention in Beweis stellen. In einem Vorher-Nachher-Vergleich bestünde hier die Gefahr, die beträchtliche Abnahme auf der Skala Depressivität irrtümlicherweise der Intervention zuzuschreiben. Das vorliegende Beispiel zeigt aber, dass Versuchs- und Kontrollgruppe gleichermaßen profitieren und die Verringerung der Depressivität auf andere Faktoren zurückzuführen ist.

(6) Kombarakaran et al. (2008) begleiteten und untersuchten ein sechs Monate dauerndes Coaching-Programm für 114 Führungskräfte einer großen, multinationalen Gesellschaft. Ziel war die Unterstützung der Führungskräfte nach einer wichtigen Aquisition. Das Coaching sollte (1) dazu beitragen, Stärken auszubauen und blinde Flecken zu erkennen, (2) die Zusammenarbeit zwischen den Führungskräften und ihren Unterstellten stärken, und es sollte (3) ihnen dabei helfen, die eigene Coaching-Kompetenzen zu entwickeln. Um diese Ziele zu erreichen, wurden 42 erfahrene Coaches für jeweils 12 Sitzungen pro Führungskraft verpflichtet. Zeitpunkt und Inhalt der Treffen sowie die Art der Kommunikation (Face-to-face, E-Mail, Telefon) wurden individuell vereinbart. Die Evaluationsinstrumente wurden, passend zum Kontext, selbst entwickelt und enthielten in der Version für die Führungskräfte 62 geschlossene (5-stufige Likert-Skalen) und 3 offene Fragen und 34 geschlossene und 22 offene Fragen in Version für die Coaches. Der Rücklauf betrug 91\% (Führungskräfte) und 69\% (Coaches). Die durchschnittliche 
Bewertung einiger Items fiel recht hoch aus. Die offenen Fragen wurden einer Inhaltsanalyse unterzogen und dazu verwendet, die Resultate der quantitativen Fragen zu validieren. Um die Vielfalt der Antworten zu reduzieren, wurde eine Hauptkomponentenanalyse des Fragebogens für die Führungskräfte durchgeführt. Die fünf extrahierten Faktoren erhielten die folgenden Bezeichnungen: Führen von Menschen, Beziehungen zu Managern, Verbesserte Zielsetzung und Prioritäten setzen, Engagement und Produktivität, Effizientere Kommunikation. Von der Anlage her verfolgen Kambarakaran et al. (2007) einen durchaus interessanten Ansatz. Leider ist die Herleitung der Resultate widersprüchlich und kaum nachvollziehbar. Angaben zu den Mittelwerten der Items fehlen. Des Weiteren fehlen Angaben dazu, worauf die immer wieder im Text gezeigten Prozentangaben Bezug nehmen. So berichten $91 \%$ der Teilnehmenden von verbesserten Führungsfähigkeiten. Aus dem Text wird nicht klar, ob sich diese Aussage auf ein einzelnes Item oder die in der Faktorenanalyse extrahierte Dimension „Führung“ bezieht. Unklar bleibt auch, auf welche Stufen (nur die höchste?) oder Stufenzusammenfassungen der fünfstufigen Skalen sich die Prozentangaben beziehen. Für die Faktorenanalyse wurden offensichtlich nicht alle Items verwendet. Eine Begründung für dieses Vorgehen fehlt.

(7) Angeregt durch die Arbeiten von Anderson (2001, 2004) und Phillips \& Schirmer (2005) integrierten Künzli \& Rietiker (2009) einen Prozess zur ROI-Schätzung in die noch laufende Standardevaluation des Coachingangebotes eines Finanzdienstleisters. Neben der quantitativen Vorher-Nachher-Erhebung wird drei Monate nach jedem Coaching eine telefonische Nachbefragung durchgeführt. Das Gespräch dauert im Schnitt eine Viertelstunde. Im Zentrum steht die Selbsteinschätzung der eigenen Leistungsfähigkeit vor und nach dem Coaching, wobei die Leistungsfähigkeit vor dem Coaching auf $100 \%$ gesetzt wird. Diese Selbsteinschätzung wird durch die konkrete und verhaltensnahe Schilderung eines oder mehrerer kritischer Ereignisse validiert. So schätzte z. B. ein Bereichsleiter die Verbesserung seiner Produktivität für die Organisation auf $10 \%$. Die Schilderung des Ereignisses förderte zutage, das er vor dem Coaching sehr viele Überstunden angehäuft hatte, die nicht mehr kompensiert werden konnten. Während des Coachings setzte er sich intensiv mit seinen Delegationsfähigkeiten auseinander und ist heute wieder in der Lage, die normalen Arbeitszeiten einzuhalten. Obwohl die Überstunden nicht erfasst wurden, erscheint der Wert einer 10\%-igen Produktivitätssteigerung (ca. 4 Stunden pro Woche) plausibel. Bei einem Vollkostensatz von CHF 600.- für eine Coachingstunde (inkl. Opportunitätskosten und Prozesskosten), einer Coachingdauer von 12 Stunden und Lohnvollkosten von CHF 150.000.- ergibt sich eine Rendite von mehr als 200\%. Insgesamt ergab sich aus den bisher geführten 13 Interviews eine durchschnittliche Leistungssteigerung von 10,5\%. Bei einer durchschnittlichen Coachingdauer von gut 12 Stunden und den oben aufgeführten Vollkostensätzen für das Coaching sowie den Lohn ergibt sich somit Rendite von ca. 270\%. Auch wenn die Schilderungen der Verhaltensänderungen das Ausmaß der Leistungssteigerungen plausibel erscheinen lassen, sind solche Schätzungen aufgrund der fehlenden Vergleichsgruppe und der Subjektivität der Einschätzung mit Vorsicht zu interpretieren.

(8) Als Einzelfallstudie fällt die Arbeit von Orenstein (2006) ein wenig aus dem Rahmen der Eingangs formulierten Ein- und Auschlusskriterien. Orenstein verwendete ein sehr aufwändiges, hoch individualisiertes, aber valides Evaluationsdesign für einen einzelnen Kunden. Die Bewertung des Coachingerfolgs erfolgte in Form eines $360^{\circ}$-Feedback 
durch 20 Personen verschiedener Hierarchiestufen auf der Basis des Empathic Organic Questionnaires von Alderfer \& Brown (1972). Dieses ist kein Fragebogen im eigentlichen Sinne, sondern ein Prozess zur Konstruktion eines solchen. Vor der Konstruktion des Fragebogens wurden Coaching-Ziele aus den drei vorher identifizierten Handlungsfeldern Motivation, Kommunikation und interpersonelle Kompetenzen abgeleitet: Die Führungskraft wollte die Anerkennung der Mitarbeitenden gewinnen und zugänglicher, fürsorglicher und geduldiger werden, und sie wollte lernen, ihre Mitarbeitenden nicht mehr in der Öffentlichkeit zur kritisieren oder sie einzuschüchtern. Auf der Basis der Zielformulierung führte Orenstein qualitative Interviews mit 15 Peers der Führungskraft durch. Aus diesen 15 Interviews wurden in der Folge 40 Aussagen herausgefiltert und noch einmal anhand der Ziele auf Passung überprüft. Im folgenden Schritt wurden die Items drei Kategorien zugeordnet: 19 Items, die in direktem Zusammenhang mit den Coachingzielen standen (Beispiel-Item: „He belittles me“) und 11 Items, die mutmaßlich nur in mittelbarem Zusammenhang mit den Coachingzielen standen (Beispiel-Item: „He behaves in unpredictable ways"). Um die Validität der Befragung zu erhöhen, wurden zusätzlich 10 weitere Items gebildet, die mutmaßlich in keinem Zusammenhang mit den Coachingzielen standen (Beispiel-Item: „He gets the job done“). Erwartet wurde, dass sich die Bewertungen der Items der ersten Kategorie am deutlichsten verändern würden. Auf den Items der zweiten Kategorie wurden mäßige Veränderungen und von denjenigen der dritten Kategorie keine Veränderungen erwartet. Von den 19 Items, die durch Coaching beeinflusst werden sollten, veränderten sich 15 signifikant in die gewünschte Richtung. Von den 11 in mittelbarem Zusammenhang mit den Zielen stehenden Items veränderten sich 4 signifikant. In der dritten Kategorie (10 Items), wurde erwartungsgemäß keine Veränderung festgestellt, was für die Validität der Methode spricht.

\section{Zusammenfassung, offene Fragen und Folgerungen}

\subsection{Zusammenfassung}

Die theoretische Verankerung ist nach wie vor sehr heterogen. Zwar wird inzwischen in den meisten Arbeiten der Coachingbegriff definiert. Dies ist hilfreich, ist es doch oft schwierig, zu entscheiden, worum es sich bei der besprochenen Intervention handelt. Nach der Begriffsdefinition werden die theoretischen Bezüge oft schwächer. Instrumente werden ad hoc entwickelt, die dahinter stehenden Konstrukte kaum expliziert. Diese individuellen Lösungen sind häufig dem Kontext und dem Zweck angepasst, erschweren aber die Einordnung in einen größeren Kontext. So beschreiben z. B. Alvey \& Barcley (2007) die Entwicklung von Vertrauen in der Coaching-Beziehung. Das Zielkonstrukt selbst, das Vertrauen, bleibt ungeklärt. Vermutlich setzen die Autorinnen ein gemeinsames Verständnis von Vertrauen in der Zielgruppe voraus. Evers et al. (2008) berufen sich auf die gut beschriebenen Konzepte Selbstwirksamkeit und Ergebniserwartungen von Bandura (1986), entwickeln aber eine eigene Operationalisierung. Wie valide diese ist, kann nicht überprüft werden.

Bowles et al. (2007) lehnen sich bei der Konstruktion des vermuteten Wirkfaktors an den Begriff der ,,adherence“ (Meichenbaum \& Turk 1987: 20) an; wie valide ihre Opera- 
tionalisierung ist, lässt sich nicht überprüfen. Orenstein (2006) wählt eine ideografische Vorgehensweise und entwickelt das Evaluationsintrument direkt aus dem Kontext heraus. Dies ist selbstverständlich eine angepasste Lösung, die theoretische Anschlussfähigkeit geht so aber verloren, und Art und Ausmaß der Wirkungen sind schwer einzuordnen. Eine ähnliche Vorgehensweise wählen Kamborakaran et al. (2008). Auch hier wird das Instrument aus dem Evaluationsprozess entwickelt. Durch die Faktorisierung der Items entsteht ein bereichsspezifsches Kompetenzmodell, dessen Dimensionen aber theoretisch nicht anschlussfähig sind. Einzig Evers et al. (2006) verwenden mit dem DASS-21 ein validiertes und theoretisch hinterlegtes Instrument. Bowles et al. (2007) und Künzli \& Rietiker (2008) beziehen sich auf ,objektive“ Leistungsindikatoren und kommen, abgesehen von Coaching-Definitionen, ohne weitere theoretische Bezüge aus.

Die valide Messung der Wirksamkeit einer Intervention ist eng mit der Verwendung des Designs der randomisierten Kontrollgruppenstudie verbunden. Nur so lassen sich Wirkungen kausal mit der Intervention verbinden. Unter den 8 besprochenen Arbeiten verwenden immerhin drei ein quasiexperimentelles Design (Bowles 2008; Evers et al. 2006; Gyllensten \& Palmer 2005). Die anderen fünf Arbeiten sind qualitativer Art oder Querschnittsmessungen, was die kausale Verbindung der Wirkungen mit dem Coaching in Frage stellt. Multiperspektivisch ist nur eine Untersuchung (Orenstein 2006) angelegt. Sie lässt die Zielerreichung nicht nur durch den Klienten selbst, sondern auch durch seine Peers bewerten. Für die Nachhaltigkeit von Coaching gibt es keine neuen Belege. Keine der 8 Untersuchungen verwendet mehr als zwei Messzeitpunkte. Bisher sind mir nur zwei Studien mit drei Messzeitpunkten bekannt (Rohmert \& Schmid 2003; Finn, Mason \& Griffin 2006). Hier besteht noch großer Bedarf. Nach wie vor werden sehr vielfältige Indikatoren für die postulierten Wirkfaktoren und Wirkungen verwendet. In den 8 Untersuchungen werden 7 unterschiedliche Indikatoren verwendet. Nur Anderson (2004) und Künzli \& Rietiker (2008) verwenden mit der Rendite die gleiche Ergebnisgröße. Nur in einer Studie (Gyllensten \& Palmer 2005) wird mit dem DASS-21 ein validiertes Instrument zur psychischen Gesundheit verwendet. Es konnten aber keine signifikanten Veränderungen gefunden werden. Dieses Resultat ist konsistent mit demjenigen von Spence \& Grant (2005) aus einer Stichprobe von 64 über die Medien gewonnen Personen. Auch dort konnten auf dem DASS-21 keine signifikanten Veränderungen festgestellt werden. Dies ist ernüchternd, geht man doch davon aus, dass Coaching eine stressvermindernde Intervention ist. Evers et al. (2006) arbeiten mit einem eigenen Instrument zur Messung der bereichsspezifischen Selbstwirksamkeits- und Ergebniserwartung. Geht man davon aus, dass Coaching eher klärungs- als verhaltensorientiert arbeitet (Riedel 2003), so erscheint die Konstruktion eines Instruments zur Erhebung unterschiedlicher Erwartungstypen sehr einleuchtend. Da es aber ad hoc entwickelt und noch nicht überprüft wurde, ist die Validität in Frage zu stellen. Zudem ist es schwer fassbar, da keine Beispiel-Items genannt wurden.

$\mathrm{Ob}$ und inwiefern sich die eingesetzten Coaching-Ansätze inhaltlich unterscheiden, kann aufgrund der Texte nicht bestimmt werden. Zu unbestimmt bleiben die Beschreibungen der verschiedenen Herangehensweisen. Nur eine Arbeit (Evers et al. 2006) enthält mit dem Verweis auf das GROW-Modell einen expliziten Bezug zu einem bestimmten Coaching-Ansatz. Dementsprechend kann auch die Frage, ob sich die eingesetzten Methoden hinsichtlich Wirksamkeit unterscheiden, aus Texten nicht hergeleitet werden. 
Für Effektstärkenvergleiche gibt es noch zu wenige Studien, und es fehlen die Voraussetzungen. Kontextfaktoren, wie z. B. das Organisationsklima, werden nirgends thematisiert. Dies ist bedauernswert, da immer wieder betont wird, wie wichtig ein unterstützendes Klima oder die Vorgesetzten für den Erfolg von Coaching seien. Coaching geschieht in einem ökomischen Kontext. Betont wird daher die Bedeutung von Leistungssteigerungen oder die Verbesserung der Produktivität durch Coaching. Am überzeugendsten sind hier die Resultate von Bowles et al. (2007), ihm gelingt der Nachweis einer eindeutigen Leistungssteigerung unter einem quasiexperimentellen Design. Leider wurden die Daten der Interventions- und der Kontrollgruppe keinem Matchingverfahren unterzogen. Auch die Arbeiten von Kambarakaran et al. (2008), Anderson (2004) und Künzli \& Rietiker (2009) befassen sich mit Produktivität und der Rendite von Coaching. Alle drei verwenden aber retrospektive resp. qualitative Designs ohne Vergleichsgruppen, und die Daten beruhen auf Selbstratings. Der Erfolgsnachweis beruht damit bestenfalls auf Plausibilität.

\subsection{Offene Fragen}

Obwohl hier neue Evidenz für die Wirksamkeit von Coaching vorgestellt wurde, bleiben mehr offene als beantwortete Fragen übrig. Unbeantwortet bleiben die meisten von Greif (2008: 277) in seinem Strukturmodell aufgeworfenen Fragen zu den Voraussetzungen, den Wirkfaktoren und den Wirkungen: Welche Bedeutung hat die Veränderungsmotivation und die Reflexivität des Klienten? Welche Rollen spielt die Affektreflexion und -Kalibrierung während der Beratung? Wie förderlich ist die Unterstützung der ergebnisorientierten Problem- und Selbstreflexion für das Ergebnis? Was ist die Wirkung und der Nutzen von Verlaufsevaluationen? Inwiefern trägt Coaching zur Potenzial- und Selbstentwicklung bei?

Noch keine Untersuchung widmet sich dem Einfluss von förderlichen oder hinderlichen Einflüssen aus dem Kontext. Wie bedeutsam ist die Unterstützung durch Vorgesetzte? Inwiefern spielen Organisations- oder Teamklima eine Rolle? Auch die Datenlage zu verordneten Coachings ist noch sehr dünn. Erste Hinweise dazu findet man bei Brauer (2004). Sie fand keine Korrelation zwischen Freiwilligkeit und Zielerreichung. Zum Thema „nicht erfolgreiches Coaching“ gibt es erst zwei Arbeiten (Krebs 2007; Mellman 2007), allerdings an studentischen Stichproben. Noch keine Arbeit nimmt sich des Themas der Unbedenklichkeit an. Wenn Coaching wirkt, kann es auch schaden. Ob das Thema „schädliches Coaching“ ein Tabuthema ist oder schlicht nicht vorkommt, kann nicht beantwortet werden. M.E. wird es in Zukunft aber unerlässlich sein, sich auch diesem Thema zu stellen. Ähnlich verhält es sich mit den Themen Indikation sowie relative und kombinierte Wirksamkeit von Coaching. Bei welchen Problemstellungen und Anliegen ist Coaching die Methode der Wahl? Wann ist Coaching die effektivere Maßnahme verglichen z.B. mit Training? Eine vergleichende Studie zwischen Training und Coaching ist mir nicht bekannt. Wann ist eine Kombination von Training und Coaching die beste Wahl? Betrachtet man z. B. nur die Kosten, ist Coaching im Vergleich zu Training eine eher teure Maßnahme. Unter Einbezug der Nutzenseite könnte sich dieses Verhältnis aber verschieben. Übersichtsarbeiten zum Thema Training zeigen, dass ihr Wirkungsgrad eher gering ist (Baldwin \& Ford 1988). Eine frühe Untersuchung von Greif \& Scheidewig (1996) zu einer kombinierten Maßnahme von Training und Coaching zeigte, dass durch 
Transfercoaching 90-100\% der Lernziele umgesetzt wurden. Kombinationen von Coaching mit $360^{\circ}$-Feedback werden bei Thach (2002) und Smither et al. (2003) besprochen. Dies sind Fragen, mit denen sich Praktiker schon längere Zeit befassen. Seitens der empirischen Forschung sind hier aber kaum Beiträge ausfindig zu machen.

Meines Wissens widmet sich erst eine Arbeit (Behrendt 2004) dem Vergleich unterschiedlicher Coachingansätze. Er vergleicht systemisches und psychodramatisches Coaching. Erste Hinweise existieren bezüglich der unterschiedlichen Wirksamkeit von angeleitetem Selbstcoaching vs. Einzelcoaching (Offermanns 2004) im Führungskräfte-Coaching. Das Einzelcoaching schnitt nur bei der Zufriedenheit besser ab. Für die Zielerreichung und die Einschätzung des praktischen Nutzens hingegen konnten keine Unterschiede gefunden werden. Zur Fragestellung der Effektivität von Peer- und Co-Coaching existiert erste ein Studie (Green, Oades \& Grant 2005). Die Stichprobe setzte sich aber nicht aus Führungskräften zusammen. Auch auf einer hierarchisch tieferen Ebene sind noch Fragen offen. Diese betreffen z. B. die Zeitabhängigkeit der Wirkungen. Wann können welche Ergebnisindikatoren sinnvoll erhoben werden? Gerade bei Veränderungen von Kompetenzen oder in der Arbeit mit hartnäckigen Problemen dürfte dies keine triviale Frage sein. Anschließend an die Frage nach dem richtigen Zeitpunkt ist die Frage nach dem richtigen Indikator bzw. dem angemessenen Instrument zu stellen. Erste Antworten hierzu findet man bei Greif (2008) und Grant (2001, 2003, 2007, 2008).

\subsection{Schlussfolgerungen}

Noch vor wenigen Jahren ließ ich an einer Veranstaltung den Satz fallen, wer sich mit Coaching-Forschung befasse, bewege sich in einer Wüste. Unterdessen stelle ich mit Freude fest, dass die Wüste lebt und verschiedene Oasen entstanden sind. Diese Verdichtungskerne sind verbunden mit den Namen Anthony M. Grant, Inhaber des Lehrstuhls für Coaching in Sydney, Siegfried Greif, emer. Prof. für Arbeits- und Organisationspsychologie, Dianne Stober an der Fielding University in St. Barbara, CA und Eric de Haan am Ashridge Center für Coaching, um hier nur einige zu nennen.

Die absolute Anzahl der Arbeiten ist aber immer noch sehr gering. Zudem gleicht Coachingforschung einem Feuchtbiotop: Auf kleinem Raum herrscht enorme Vielfalt, und die einzelnen Arbeiten nehmen wenig aufeinander Bezug. Von einem gemeinsamen Projekt „Coachingforschung“ kann noch nicht die Rede sein. Ein Grund dafür ist sicher der Zugang zum Feld. Dieser ist nach wie vor schwierig. Weder Coaches noch ihre Klienten lassen sich gerne in die Karten schauen. Dass dem so ist, mag teilweise daran liegen, dass Forschung von Praktizierenden für ihre Arbeit mit Klienten als wenig hilfreich wahrgenommen wird. Forschungen sind in der Regel auf den Nachweis mittlerer Veränderungen angelegt. Mittelwerte liefern aber keine brauchbaren Hinweise für die Einzelfallarbeit. Im Blick von Coaches ist aber immer der einzelne Klient. Ob eine Intervention im Allgemeinen wirkt, wird zwar meistens gerne vermerkt, aber kaum als hilfreich oder nützlich wahrgenommen. Wesentlich bedeutsamer für Praktizierende ist die Frage, ob die von ihnen gewählten Interventionen für den anwesenden Klienten wirksam sind oder nicht. $\mathrm{Zu}$ fordern ist eine klientenorientierte Forschung (vgl. Künzli \& Stulz, in Vorbereitung), die Aussagen und Feedbacks auf der Ebene des Einzelfalls ermöglicht, welche unmittelbar für die Beratungsarbeit genutzt werden können. Die Stellungnahme für eine klienten- 
orientierte Forschung soll aber nicht als Plädoyer gegen die Kontrollgruppenforschung verstanden werden, sondern als ein Weg in Richtung engere Zusammenarbeit zwischen Forschung und Praxis. Coaching braucht beides, die prüfende und die verbessernde Funktion der Forschung. Ein sowohl-als-auch ist nicht nur möglich, sondern auch notwendig.

\section{Literatur}

Alderfer, C.P., Brown, L.D. (1972). Designing an „empathic questionnaire“ for organizational research. Journal of Applied Psychology, 56, 456-460.

Alvey, S., Barcley, K. (2007). The characteristics of dyadic trust in executive coaching. Journal of Leadership Studies, Volume 1, 1, 18-27.

Anderson, M.C. (2004). Executive Briefing: The business impact of Leadership Coaching at a professional Services Firm. MetrixGlobal, LLC. Retrieved 22.01.2009. http://www.cylient. com/images/pdfs/MetrixGlobalROIofCoachingProfSvsExecBrief.pdf.

Anderson, M.C., Brill, P., Lynch, J. (2007). The Utilization an Impact of Leadership Coaching in Organzations: Results from the Second Annual Benchmark Study. Metrix Global LLC. Retrieved 22.01.2009 from http://www.cylient.com/ resources/2ndAnnualCoachinginOrg BenchmarkStudy2007final.pdf.

Baldwin, Ford (1988). Transfer of Training: A review and directions for future research. Personnel Psychology, 41, 63-105.

Bandura, A. (1977). Self-efficacy: Toward a unifiying theory of behavioral change. Psychological Review, 84, 191-215.

Bandura, A. (1986). Social foundations of thought and action: A social cognitive theory. Englewood Cliffs, NJ: Prentice Hall.

Behrendt, P. (2004). Was kann Psychodrama zu einem erfolgreichen Coaching beitragen? Eine wirkfaktorenorientierte Prozessanalyse im Transfercoaching. Unveröff. Diplomarbeit an der Universität Freiburg i.Br.

Bowles, S., Cunningham, J.L., De La Rosa, G.M., Picano, J. (2007). Coaching leaders in middle and executive managment: Goals, performance, Buy-in. Http://www.emaraldinsight.com/ Insight/ViewContentServlet;jsessionid=8C370D1929B719AC1E2C2560962CBF10? content Type $=$ Article $\&$ Filename $=$ Published/EmeraldFullTextArticle $/$ Articles $/ 0220280501 . \mathrm{htm}$.

Böning, U., Fritschle, B. (2005). Coaching fürs Business. Bonn: managerSeminare.

Bose, D. von, Martens-Schmidt, K., Suchardt-Hain (2003). Führungskräfte im Gespräch über Coaching. Eine empirische Studie. In: K. Martens-Schmidt (Hrsg.), Coaching als Beratungssystem. Grundlagen, Konzepte, Methoden (S. 1-54). Heidelberg: Economica.

Brauer, Y. (2004). Coaching: Eine empirische Untersuchung zu Zielsetzugn, Intervention und Coachingerfolg bei Einzelcoaching. Unveröff. Diplomarbeit an der Universität Mannheim, Lehrstuhl Psychologie I.

Dingman, M. (2004). The effects of executive coaching on job-related outcomes (Doctoral Dissertation, Regent University). Dissertational Abstracts International, AAT 3141539.

Dopfer, W. (2000). Die Führungskraft als Coach: Erfolgsfaktor im Vertrieb. Der erfolgreiche Einsatz des „Coaching“ in der Mitarbeiterführung verlangt eine Klärung und Operationalisierung des Modewortes. In: HypoVereinsbank - Bereich Bayern-Süd, München (Hrsg.), Personalführung, 33 (4), S. 60-64.

Dzierzon, S.A. (2004). Personzentriertes Beziehungsverhalten beim Coaching. Unveröff. Magisterarbeit (gekürzte Fassung), FernUniversität.

Ellinger, A.D., Ellinger, A.E., Keller, S.B. (2003). Supervisory Coaching Behavior, Employee Satisfaction, and Warehouse Employee Performance. A Dyadic Perspective in the Distribution Industry. Human Resource Development Quaters, 14 (4), p. 435-458. 
Evers, WJ.G., Browers, A., Tomic, W. (2006). A quasi-experimental Studiy on Management Coaching Effectiveness. Consulting Psychology Jorunal: Practice and Research, 58, 3, 174-182.

Finn, F., Mason, C. \& Griffin, M. (2006). Investigating change over time - The effects of executive coaching on leaders' psychological states and behavior. Paper held at the 26th International Congress of Applied Psychology, July 16-21, 2006 Athens, Greece.

Gyllenstein, K., Palmer, S. (2005). Can Coaching Reduce Workplace Stress? A Quasi-Experimental Study. International Journal of Evidence Based Coaching and Mentoring, 3 (2), p. 75-85.

Gegner, C. (1997). Summary of executive coaching research project [On-line]. Available: http:// www.coachingnetwork.org.uk/ResourceCentre/Articles/ViewArticle.asp?artId=61

Grant, A.M. (2001). Towards a Psychology of Coaching. Paper presented at the Fourth Annual Oxford School of Coaching and Mentoring Conference, Heythrop Park, Oxford, UK.

Grant, A.M. (2003). The impact of Life Coaching on Goal Attainment, Metacognition and Mental Health. Social Behavior and Personality, 3 (3), p. 253-264.

Grant, A.M. (2005). Workplace, executive and life coaching: An annotated bibliography from the behavioral science literature. Unpubl. Paper University of Sydney, Coaching Psychology Unit, anthonyg@psych.usyd.edu.au.

Grant, A.M. (2007). Workplace, executive and life coaching: An annotated bibliography from the behavioral science literature (July 2007). Coaching Psychology Unit, University of Sydney.

Grant, A.M. (2008). Personnal life coaching for coaches-in-training enhances goal-attainment, insight and learning. Coaching: An international Journal of Theory, Research and Practice, $1(1), 54-70$.

Green, S., Oades, L.G., Grant, A.M. (2005). An Evaluation of a life-coaching group program: Initial fndings from a waitlist control study. In: M. Cavanagh, A.M. Grant, T. Kemp (Eds.), Evidence based coaching vol. 1: Theory, research and practice from the behavioral sciences (pp. 127141). Bowen Hills Qld: Australian Academic Press.

Greif, S. (2008). Coaching und ergebnisorientierte Selbstreflexion. Göttingen: Hogrefe.

Greif, S., Scheidewig, V. (1997). Selbstorganisiertes Lernen von Schichtleitern. In S. Greif, H.-J. Kurtz (Hrsg.), Handbuch Selbstorganisiertes Lernen (S. 283-300). Göttingen: Hogrefe.

Heckhausen, H., Gollwitzer, P.M., Weinert, F.E. (1987). Jenseits des Rubikon: Der Wille in den Humanwissenschaften. Berlin: Springer.

Hess, T., Roth, W.L. (2001). Professionelles Coaching. Heidelberg: Asanger.

Hernez-Broome (2004). Impact of coaching following a leadership development program: Coaching is key to continued development. Paper presented at the Second ICF Coaching Research Symposium, Quebec Canada.

Jansen, A., Mäthner, E., Bachmann, Th. (2004). Erfolgreiches Coaching. Wirkfaktoren im Einzelcoaching. Kröning: Asanger.

Kambarakoran, F.A., Yang, J.A., Baker, M.N., Fernandes, P.B. (2008). Executive Coaching: It works! Consulting Psychology Journal: Practice and Research, 60 (1), 78-90.

Kaufel, S., Scherer, S., Scherm, M., Sauer, M. (2006). Führungsbegleitung in der Bundeswehr - Coaching durch militärische Führungskräfte. In: W. Backhausen, J.-P. Thommen (Hrsg.), Coaching, durch systemisches Denken zur innovativen Personalentwicklung (S. 419-438). Wiesbaden: Gabler.

Krebs, K. (2007). Erfolg beim Coaching. Pilotuntersuchung zur Erprobung eines neuen Instruments zur summativen Evaluation von Coaching. Diplomarbeit im Fachgebiet Arbeits- und Organisationspsychologie, Universität Osnabrück.

Künzli, H. (2005). Wirksamkeitsforschung im Führungskräfte-Coaching. OSC, 12 (3), 231-243.

Künzli, H., Rietiker, J. (2009). Return on Investment im Führungskräfte-Coaching. Unveröff. Bericht aus dem Departement P, Zürcher Hochschule für Angewandte Wissenschaften.

Künzli, H., Stulz, N. (2009). Klientenorientierte Forschung im Coaching. In Vorbereitung.

Lovibond, P.F., Lovibond, S.H. (1995). Manual for the depression, anxiety stress scales. Sydney: Psychological Foundation. 
Meichenbaum, D., Turk, D.C. (1987). Facilitating Treatment adherence: A practioner's Guidebook. New York: Plenum Press.

Mellmann, M. (2007). Qualitative Untersuchung zur summativen Evaluation von Coaching. Diplomarbeit im Fachgebiet Arbeits- und Organisationspsychologie, Universität Osnabrück.

Offermanns, M. (2004). Braucht Coaching einen Coach? Eine evaluative Pilotstudie. Stuttgart: Ibidem.

Olivero, G., Bane, D.K., Kopelmann, R.E. (1997). Executive coaching as a transfer of training tool: Effects on productivity in a public agency. Public Personnel Management, 26 (4), p. 461-469.

Orenstein, R.L. (2006). Measuring Executive Coaching Efficacy? The Answer Was Right Here All the Time. Consulting Psychology Journal: Practice and Research, 58 (2), 106-116.

Orlinsky, D.E., Howard, K.I. (1986). Process and Outcome in Psychotherapy. In: S.L. Garfield, A.E. Bergin (Eds.), Handbook of Psychotherapy and Behavior Change (pp. 311-384). New York: Wiley.

Phillips, J.J., Schirmer, F. (2005). Return on Investment in der Personalentwicklung. Berlin: Springer.

Riedel, J. (2003). Coaching für Führungskräfte: Erklärungsmodell und Fallstudien. Wiesbaden: Deutscher Universitätsverlag.

Rohmert, E., Schmid, E.W. (2003). Coaching ist messbar. Ist Corporate Coaching eine sinnvolle Investition in Führungskräfte? New Management, H. 1-2, 46-53.

Runde, B., Bastians, F. (2005). Internes Coaching bei der Polizei NRW - eine multimodale Evaluationsstudie. Beitrag zum Coaching-Kongress 4.-5.3.2005, Frankfurt/M.

Sauer, M., Scherer, S., Scherm, M., Kaufel, S. (2004). Führungsbegleitung in militärischen Organisationen: Konzepte und erste Effekte in der Praxis. Personalführung, 11, 44-51.

Smither, J.W., London, M., Flautt, R., Vargas, Y., Kucine, I. (2003). Can working with an executive coach improve multisource feedback ratings over time? A quasi-experimental field study. Personnel Psychology, 56 (1), 23-44.

Spence, G.B., Grant, A.M. (2005). Individual and group life-coaching: Initial findings from a randomised, controlled trial. In: M. Cavanagh, A.M. Grant, T. Kemp (Eds.), Evidence based Coaching, vol 1, Theory, research and practice from the behavioral sciences (S. 145-158). Bowen Hills Qld: Australien Academic Press.

Steinmetz, B. (2005). Stressmanagement für Führungskräfte. Hamburg: Kovac.

Sue-Chan, C., Latham, G.P. (2004). The Relative Effectiveness of External, Peer, and Self-Coaches. Applied Psychology: An International Review, 53 (2), 260-278.

Thach, E.C. (2002). The impact of executive Coaching and 360 feedback on leadership effectiveness. Leadership \& Organization Development Journal, 23 (4), 205-214.

Wagemann, R. (2001). How Leaders Foster Self-Managing Team Effectivneness: Design Choces Versus Hands-on Coaching. Organization Science, 12 (5), 559-577.

Willms, J.-F. (2004). Coaching zur Umsetzung persönlicher Ziele. Entwicklung, Durchführung und Evaluation. Diplomarbeit im Fachgebiet Arbeit- und Organisationspsychologie der Universität Osnabrück.

Whitmore, J. (2002). Coaching for performance: GROWing People, Perfomance and Purpose. London: National Book Network.

Withworth, L., Kimsey-House, H., Sandahl, P. (1998). Co-active coaching: New skills for coaching people towards success in work and life. Palo Alto: Davies-Black Publishing. 


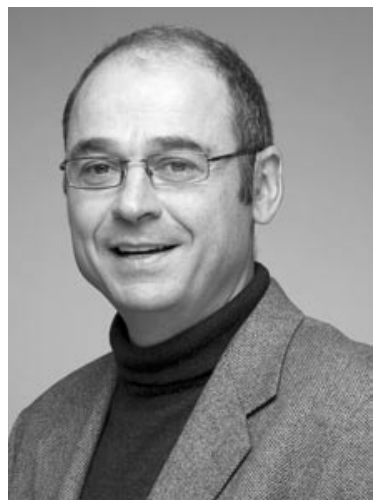

Prof. Hansjörg Künzli, geb. 1962, ist Dozent im Masterstudiengang ,Angewandte Psychologie ${ }^{6}$ und leitet den Forschungsschwerpunkt ,Beratung und Training in Organisationen " an der Zürcher Hochschule für Angewandte Wissenschaften. Anschrift: Zürcher Hochschule für Angewandte Psychologie, Minervastrasse 30, Postfach, CH-8032 Zürich, E-Mail: hansjoerg.kuenzli@zhaw. ch; www.psychologie.zhaw.ch 